\title{
Deformation quantization and Borel's theorem in locally convex spaces
}

\author{
by
}

Miroslav Engliš (Praha and Opava) and Jari Taskinen (Helsinki)

\begin{abstract}
It is well known that one can often construct a star-product by expanding the product of two Toeplitz operators asymptotically into a series of other Toeplitz operators multiplied by increasing powers of the Planck constant $h$. This is the BerezinToeplitz quantization. We show that one can obtain in a similar way in fact any starproduct which is equivalent to the Berezin-Toeplitz star-product, by using instead of Toeplitz operators other suitable mappings from compactly supported smooth functions to bounded linear operators on the corresponding Hilbert spaces. A crucial ingredient in the proof is the generalization, due to Colombeau, of the classical theorem of Borel on the existence of a function with prescribed derivatives of all orders at a point, which reduces the proof to a construction of a locally convex space enjoying some special properties.
\end{abstract}

1. Introduction and background. Let $\Omega$ be a domain in $\mathbb{C}^{n} \cong \mathbb{R}^{2 n}$ equipped with a Poisson bracket $\{\cdot, \cdot\}$, i.e. a first order bidifferential operator

$$
\{f, g\}(x)=\sum_{j, k=1}^{2 n} B_{j k}(x) \frac{\partial f}{\partial x_{j}} \frac{\partial g}{\partial x_{k}}, \quad x \in \mathbb{R}^{2 n},
$$

where, for each $x \in \Omega$, the matrix $B_{j k}(x)$ is skew-symmetric and nonsingular. Denote by $C^{\infty}(\Omega)[[h]]$ the ring of formal power series in a variable $h$ with coefficients in $C^{\infty}(\Omega)$. A star product on $\Omega$ is a $\mathbb{C}[[h]]$-bilinear map * : $C^{\infty}(\Omega)[[h]] \times C^{\infty}(\Omega)[[h]] \rightarrow C^{\infty}(\Omega)[[h]]$ such that

(i) $*$ is associative,

(ii) there exist bidifferential operators $C_{j}: C^{\infty}(\Omega) \times C^{\infty}(\Omega) \rightarrow C^{\infty}(\Omega)$

2000 Mathematics Subject Classification: Primary 46A13; Secondary 53D55, 26B05, $47 \mathrm{~B} 35$.

Key words and phrases: Berezin-Toeplitz quantization, Borel theorem, Fréchet space, inductive limit.

Research of the first author supported by GA ČR grant no. 201/03/0041 and by AV ČR IRP no. AV0Z10190503. 
such that

$$
f * g=\sum_{j=0}^{\infty} h^{j} C_{j}(f, g), \quad \forall f, g \in C^{\infty}(\Omega) ;
$$

(iii) the operators $C_{j}$ satisfy

$$
\begin{gathered}
C_{0}(f, g)=f g, \\
C_{1}(f, g)-C_{1}(g, f)=\frac{i}{2 \pi}\{f, g\},
\end{gathered}
$$

and

$$
C_{j}(f, \mathbf{1})=C_{j}(\mathbf{1}, f)=0, \quad \forall j \geq 1 .
$$

Note that the last equality means precisely that $\mathbf{1}$ is the unit element for $*$.

REMARK. Generalization to complex manifolds $\Omega$ is straightforward.

Two star products $*, *^{\prime}$ are called equivalent if there exists a sequence of linear differential operators $M_{0}, M_{1}, M_{2}, \ldots$ on $C^{\infty}(\Omega)$ with $M_{0}=I$ (the identity operator) such that we have the following equality of formal power series:

$$
M\left(f *^{\prime} g\right)=M f * M g, \quad \forall f, g \in C^{\infty}(\Omega)[[h]]
$$

where

$$
M f=\sum_{j=0}^{\infty} h^{j} M_{j} f .
$$

Star products are the object of study of deformation quantization, and were first introduced in the seminal paper by Bayen, Flato, Fronsdal, Lichnerowicz and Sternheimer [BF]. Some more information about them can be found e.g. in the recent surveys by Gutt $[\mathrm{Gu}]$ or by S.-T. Ali and the first author $[\mathrm{AE}]$.

Toeplitz star product. One can sometimes construct a star product using Toeplitz operators. Namely, under suitable hypotheses on $\Omega$ and the Poisson structure (the boundary behaviour of the $B_{j k}$ in (1)), there exists a family of measures $\mu_{h}$ on $\Omega, 0<h<1$, such that the following holds. Let $L_{\text {hol }}^{2}\left(\mu_{h}\right)$ be the subspace of holomorphic functions in $L^{2}\left(\mu_{h}\right)$ (weighted Bergman space), $P_{h}: L^{2}\left(\mu_{h}\right) \rightarrow L_{\text {hol }}^{2}\left(\mu_{h}\right)$ the orthogonal projection, and for $f$ a bounded continuous function on $\Omega$ define the Toeplitz operator $T_{f}^{(h)}$ on $L_{\text {hol }}^{2}\left(\mu_{h}\right)$ by $T_{f}^{(h)} \phi=P_{h}(f \phi)$. Then for any $f, g \in \mathcal{D}(\Omega)\left(:=\right.$ the functions in $C^{\infty}(\Omega)$ with compact support), there is an asymptotic expansion

$$
T_{f}^{(h)} T_{g}^{(h)} \simeq \sum_{j=0}^{\infty} h^{j} T_{C_{j}(f, g)}^{(h)} \quad \text { as } h \rightarrow 0
$$


with some bidifferential operators $C_{j}$ (independent of $f, g$ ). Further, these operators $C_{j}$ define - via the formula (2) - a star product on $\Omega$.

Here the expansion (5) is understood in the sense of operator norms, i.e. for each $N=0,1,2, \ldots$,

$$
\left\|T_{f}^{(h)} T_{g}^{(h)}-\sum_{j=0}^{N} h^{j} T_{C_{j}(f, g)}^{(h)}\right\| \leq C_{N, f, g} h^{N+1}, \quad \forall h \in(0,1) .
$$

REMARK. Formally, we can write (5) as

$$
T_{f}^{(h)} T_{g}^{(h)}=T_{f * g}^{(h)} .
$$

EXAmple ([Cob]). If $\Omega=\mathbb{C}$ and $d \mu_{h}(z)=(\pi h)^{-1} e^{-|z|^{2} / h} d z$ (where $d z$ stands for the two-dimensional Lebesgue measure), then (5) holds with

$$
C_{j}(f, g)=\frac{1}{j !} \frac{\partial^{j} f}{\partial z^{j}} \frac{\partial^{j} g}{\partial \bar{z}^{j}} .
$$

Similarly, (5) holds for $\Omega=\mathbb{D}$, the unit disc, with the standard weighted Bergman spaces corresponding to $d \mu_{h}(z)=\frac{h+1}{\pi h}\left(1-|z|^{2}\right)^{1 / h} d z[\mathrm{KL}]$.

Other situations when the Berezin-Toeplitz quantization can be carried out include bounded symmetric domains [BLU] (see also [E3] for an extension from functions in $\mathcal{D}$ to functions not necessarily having compact support), strictly pseudoconvex domains with Poisson brackets having a reasonable boundary behaviour [E1], or, provided one considers the manifold case and allows also spaces $L_{\mathrm{hol}}^{2}$ of sections of line bundles (instead of just functions), all compact Kähler manifolds [BMS], [Sch].

The Berezin-Toeplitz quantization prompts the following definition.

Definition. We say that a star product (2) is induced by operators if there exists a family of Hilbert spaces $\mathcal{H}_{h}, 0<h<1$, a "large" subspace $\mathcal{Z} \subset$ $C^{\infty}(\Omega)$, and linear maps $f \mapsto Q_{f}^{(h)}$ from $\mathcal{Z}$ into bounded linear operators on $\mathcal{H}_{h}$ such that

$$
Q_{f}^{(h)} Q_{g}^{(h)} \simeq \sum_{j=0}^{\infty} h^{j} Q_{C_{j}(f, g)}^{(h)} \quad \text { as } h \rightarrow 0, \quad \forall f, g \in \mathcal{Z},
$$

in the sense of operator norms.

Here being "large" can be interpreted, for instance, as follows:

(8) for each finite set of multiindices $\alpha_{1}, \ldots, \alpha_{k}$, complex numbers $w_{1}, \ldots, w_{k}$, and point $z \in \Omega$, there exists $f \in \mathcal{Z}$ such that

$$
D^{\alpha_{j}} f(z)=w_{j}, \quad \forall j=1, \ldots, k,
$$


where $D^{\alpha}$ denotes the operator of differentiation. The merit of (8) is that it ensures that the knowledge of $C_{j}(f, g)$ for all $f, g \in \mathcal{Z}$ already determines the $C_{j}$ uniquely.

Conjecture. Every star product is induced by operators.

At the moment, we have no idea how to attack this conjecture. But we are able to prove at least a weaker result:

MAin TheOREM. Every star product equivalent to the Toeplitz star product is induced by operators.

For the case of bounded symmetric domains and star-products which are invariant with respect to holomorphic automorphisms, this theorem was proved by one of the authors in [E2], using heavily the special machinery of Lie groups available in that setup (above all, the Helgason-Fourier transform and the related theory of invariant differential operators on symmetric spaces). No such thing is available in the general case treated here, and thus we use another approach building on an extension of the classical theorem of Borel to Fréchet spaces, due to Colombeau [Col].

The paper is organized as follows. In Section 2, we show how the proof of the theorem can be reduced to the problem of existence of a locally convex space of functions on $\Omega$ possessing certain properties. This space is then constructed in Section 3 as the inductive limit of a sequence of Banach spaces. The necessary prerequisites on locally convex spaces can be found e.g. in the books of Jarchow [Ja], Koethe [Koe], Meise and Vogt [MV], or Bonet and Perez Carreras [BnC]. In the final Section 4, we briefly mention also a nonlinear variant of the above quantization procedure, for which a much simpler proof can be given.

2. Plan of proof of Main Theorem. Let $*$ denote the Toeplitz star product, and let $*^{\prime}$ be a star product equivalent to $*$. Let $M_{0}=I, M_{1}, M_{2}, \ldots$ be the differential operators furnishing the equivalence, and let $M$ denote the linear operator on $C^{\infty}(\Omega)[[h]]$ given by (4). Writing (5) and (7) formally as

$$
T_{f} T_{g}=T_{f * g}, \quad Q_{f} Q_{g}=Q_{f *^{\prime} g},
$$

and comparing this with (3), we see that if we could take

$$
Q_{f}^{(h)}:=T_{M f}^{(h)}
$$

then we would be done. The problem is that $M f$ is just a formal power series, which may diverge if one assigns to $h$ some value. So we need to approximate, in some sense, the formal power series $M$ by genuine operators. 
Denote by $C_{j}$ the coefficients (2) of the Toeplitz star product, and by $C_{j}^{\prime}$ the coefficients of $*^{\prime}$. Expanding (3) and comparing the expressions at like powers of $h$ on both sides, we see that $C_{j}$ and $C_{j}^{\prime}$ are related by

$$
\sum_{j+k=N} M_{j} C_{k}^{\prime}(f, g)=\sum_{j+k+l=N} C_{j}\left(M_{k} f, M_{l} g\right), \quad \forall f, g \in C^{\infty}(\Omega),
$$

for each $N=0,1,2, \ldots$

Assume that we can construct a vector space $\mathcal{Z}$ contained in $C^{\infty}(\Omega)$ such that

(a) $\mathcal{D} \cap \mathcal{Z}$ is "large", in the sense of $(8)$;

(b) $M_{j} \mathcal{Z} \subset L^{\infty}$ for all $j$;

(c) $C_{l}\left(M_{j} \mathcal{Z}, M_{k} \mathcal{Z}\right) \subset L^{\infty}$ for all $j, k, l$;

(d) $C_{k}^{\prime}(\mathcal{Z}, \mathcal{Z}) \subset \mathcal{Z}$ for all $k$; and, finally,

(e) there exists a family of linear operators $M^{(h)}, 0<h<1$, from $\mathcal{Z}$ into $L^{\infty}$ such that for each $N=0,1,2, \ldots$ and $f \in \mathcal{Z}$,

$$
\left\|\left(M^{(h)}-\sum_{j=0}^{N} h^{j} M_{j}\right) f\right\|_{\infty} \leq C_{f, N} h^{N+1}
$$

with some finite constant $C_{f, N}$, for all $0<h<1$.

(Note that, as $M_{0}=I$, (b) implies in particular that $\mathcal{Z} \subset L^{\infty}$.)

Granted this, let us set, for $f \in \mathcal{Z}$,

$$
Q_{f}^{(h)}:=T^{(h)}\left[M^{(h)} f\right]
$$

where, for typographical reasons, we started writing $T^{(h)}[f]$ instead of $T_{f}^{(h)}$. Since the norm of a Toeplitz operator always satisfies

$$
\left\|T_{f}^{(h)}\right\| \leq\|f\|_{\infty}
$$

we see from (e) that, for each $N=0,1,2, \ldots$,

$$
\left\|T^{(h)}\left[M^{(h)} f\right]-\sum_{j=0}^{N} h^{j} T^{(h)}\left[M_{j} f\right]\right\|=O\left(h^{N+1}\right) .
$$

(Note that $M^{(h)} f, M_{j} f \in L^{\infty}$ in view of (e) and (b).)

If $g$ is another function from $\mathcal{Z}$, it follows that

$$
\left\|Q_{f}^{(h)} Q_{g}^{(h)}-\left(\sum_{j=0}^{N} h^{j} T^{(h)}\left[M_{j} f\right]\right)\left(\sum_{j=0}^{N} h^{j} T^{(h)}\left[M_{j} g\right]\right)\right\|=O\left(h^{N+1}\right) .
$$

Finally, if $f$ and $g$ (and hence also $M_{j} f, M_{k} g$ ) belong in addition to $\mathcal{D}$, 
then (6) applies to $T^{(h)}\left[M_{j} f\right] T^{(h)}\left[M_{k} g\right]$, for each $j$ and $k$; thus for any $f, g \in \mathcal{D} \cap \mathcal{Z}$,

$$
\begin{aligned}
Q_{f}^{(h)} & Q_{g}^{(h)}=\sum_{j, k=0}^{N} h^{j+k} T^{(h)}\left[M_{j} f\right] T^{(h)}\left[M_{k} g\right]+O\left(h^{N+1}\right) \\
= & \sum_{j, k, l=0}^{N} h^{j+k+l} T^{(h)}\left[C_{l}\left(M_{j} f, M_{k} g\right)\right]+O\left(h^{N+1}\right) \quad \text { by (6) } \\
= & \sum_{j+k+l \leq N} h^{j+k+l} T^{(h)}\left[C_{l}\left(M_{j} f, M_{k} g\right)\right]+O\left(h^{N+1}\right) \quad \text { by (c) } \\
= & \sum_{j+k \leq N} h^{j+k} T^{(h)}\left[M_{j} C_{k}^{\prime}(f, g)\right]+O\left(h^{N+1}\right) \quad \text { by (9) } \\
= & \sum_{j, k=0}^{N} h^{j+k} T^{(h)}\left[M_{j} C_{k}^{\prime}(f, g)\right]+O\left(h^{N+1}\right) \quad \text { by (d) and (b) } \\
= & \sum_{j=0}^{N} h^{j} T^{(h)}\left[M_{j} \sum_{k=0}^{N} h^{k} C_{k}^{\prime}(f, g)\right]+O\left(h^{N+1}\right) \\
= & T^{(h)}\left[M^{(h)} \sum_{k=0}^{N} h^{k} C_{k}^{\prime}(f, g)\right]+O\left(h^{N+1}\right) \quad \text { by (d) and (10) again } \\
= & Q^{(h)}\left[\sum_{k=0}^{N} h^{k} C_{k}^{\prime}(f, g)\right]+O\left(h^{N+1}\right) .
\end{aligned}
$$

(All the $O$-terms relate to errors in operator norm.) Thus (7) holds (with $C_{j}^{\prime}$ in place of $C_{j}$ ), and the proof is complete.

It thus only remains to construct the space $\mathcal{Z}$ with the above properties.

3. Construction of the space $\mathcal{Z}$. Recall that the classical theorem of Borel asserts that for any sequence $f_{n}$ of complex numbers, there exists a function $f \in \mathcal{D}(\mathbb{R})$ such that

$$
f^{(j)}(0)=j ! f_{j}, \quad \forall j=0,1,2, \ldots
$$

In particular, by Taylor's formula, it follows that

$$
\left|f(h)-\sum_{j=0}^{N} h^{j} f_{j}\right| \leq C_{f, N} h^{N+1}, \quad \forall h \in \mathbb{R} .
$$

Comparing this with the condition (e), we see that (e) is tantamount to having a Borel theorem for functions on $\mathbb{R}$ with values in the space of operators from $\mathcal{Z}$ into $L^{\infty}$ equipped with the strong operator topology (the topology of 
uniform convergence in norm on finite subsets of $\mathcal{Z}$ ). Unfortunately, it turns out that in this generality, i.e. for functions with values in a locally convex space, Borel's theorem may fail in general (see [Col]).

However, it is a notable result of Colombeau [Col] that Borel's theorem is valid for functions on $\mathbb{R}$ with values in a Fréchet space. Furthermore, it is known that if $\mathcal{Z}$ is an $(L B)$-space, that is, a countable inductive limit of Banach spaces, then the space $L_{b}\left(\mathcal{Z}, L^{\infty}(\Omega)\right)$ of continuous linear operators, endowed with its natural locally convex topology of uniform convergence on bounded sets, automatically becomes a Fréchet space. Consequently, if our $\mathcal{Z}$ is an $(L B)$-space, then Colombeau's result applies, and we get our requirement (e) granted.

Our purpose will therefore be to construct an $(L B)$-space $\mathcal{Z} \subset C^{\infty}(\Omega)$ which satisfies the conditions $(\mathrm{a})-(\mathrm{d})$.

Let $c_{j \alpha} \in C^{\infty}(\Omega)$ be the coefficients of the differential operators $M_{j}$, i.e.

$$
M_{j} f(x):=\sum_{\alpha \text { multiindex }} c_{j \alpha}(x) D^{\alpha} f(x) ;
$$

and similarly define

$$
\begin{aligned}
C_{j}\left(M_{k} f, M_{l} g\right) & =\sum_{\alpha, \beta} c_{j k l \alpha \beta} D^{\alpha} f \cdot D^{\beta} g \\
C_{k}^{\prime}(f, g) & =\sum_{\alpha, \beta} c_{k \alpha \beta}^{\prime} D^{\alpha} f \cdot D^{\beta} g .
\end{aligned}
$$

Let us enumerate the countable set $\left\{c_{j \alpha}\right\}_{j, \alpha} \cup\left\{c_{j k l \alpha \beta}\right\}_{j, k, l, \alpha, \beta} \cup\left\{c_{k \alpha \beta}^{\prime}\right\}_{k, \alpha, \beta}$ of all the above coefficients as $v_{j}, j \in \mathbb{N}$. (Here and in what follows, $\mathbb{N}:=\{0,1,2, \ldots\}$.)

LEMMA 1. There exists a $C^{\infty}$ weight function $w: \Omega \rightarrow \mathbb{R}^{+}$such that $w \geq 1, w(x) \rightarrow \infty$ as $x$ tends to the boundary or to infinity, and

$$
\gamma_{\alpha, j}:=\sup _{x \in \Omega} \frac{\left|D^{\alpha} v_{j}(x)\right|}{w(x)}<\infty
$$

for all $j \in \mathbb{N}$ and all multiindices $\alpha$.

Proof. Enumerate the (countable) set of all the functions $D^{\alpha} v_{j}, j \in \mathbb{N}$, $\alpha \in \mathbb{N}^{n}$, as $f_{k}, k=1,2, \ldots$ Further, pick a sequence $\phi_{j}$ of functions in $\mathcal{D}(\Omega)$ such that $0 \leq \phi_{j} \leq 1$, the union of the supports of $\phi_{j}$ is all of $\Omega$, and $\phi_{j+1}=1$ on the support of $\phi_{j}$. Define $K_{j}:=\operatorname{supp} \phi_{j}$, and set $f_{0}(x):=\sum_{j}\left(1-\phi_{j}\right)$. Thus $K_{j}$ is an increasing sequence of compact subsets such that the union of their interiors is $\Omega$, and $f_{0}$ is in $C^{\infty}(\Omega), f_{0} \geq 0$ and $f_{0}(x) \rightarrow \infty$ as $x$ tends to the boundary of $\Omega$ or to infinity. Now choose constants $C_{m}$ such that

$$
\sup _{x \in K_{m}, j \leq m,|\alpha| \leq m}\left|D^{\alpha} f_{j}(x)\right| \leq C_{m} .
$$


Set

$$
w(x):=1+\sum_{j=0}^{\infty} \frac{f_{j}(x)}{2^{j} C_{j}} .
$$

The sum converges uniformly on compact subsets, together with all its partial derivatives, thus $w \in C^{\infty}(\Omega)$. Clearly $w \geq 1$, and $w(x) \rightarrow \infty$ as $x$ tends to the boundary or to infinity since $w \geq C_{0}^{-1} f_{0}$. Finally, $f_{k} / w \leq 2^{k} C_{k}$. Thus $w$ does the job we need.

We fix a function $w$ as in the last lemma from now on, and also set

$$
W(x):=e^{w(x)} \text {. }
$$

Define, for $j \in \mathbb{N}$,

$$
\kappa_{j}:=\sup _{x \in \Omega} \frac{w(x)^{j}}{W(x)} .
$$

From the fact that $w \rightarrow \infty$ as $x$ tends to the boundary or to infinity it follows that each $\kappa_{j}$ is finite.

Finally, we fix from now on a sequence $\phi_{k}$ of functions in $\mathcal{D}$ such that the union of their supports is $\Omega$, and $\phi_{k+1}=1$ on the support of $\phi_{k}$.

LEMMA 2. There exists a sequence $\left\{\varepsilon_{j}\right\}_{j \in \mathbb{N}}$ such that $0<\varepsilon_{j} \leq 1$ for all j and

$$
\sup _{x \in \Omega} W(x) \sum_{\alpha} \varepsilon_{|\alpha|}\left|D^{\alpha}\left(x^{\gamma} \phi_{k}(x)\right)\right|<\infty
$$

for any $k \in \mathbb{N}$ and any multiindex $\gamma$. Here the summation extends over all multiindices $\alpha \in \mathbb{N}^{n}$, and $|\alpha|:=\alpha_{1}+\cdots+\alpha_{n}$.

Proof. Choose again constants $C_{m}^{\prime}<\infty$ such that

$$
\sup _{x \in \Omega,|\gamma| \leq m, k \leq m,|\alpha| \leq m} W(x)\left|D^{\alpha}\left(x^{\gamma} \phi_{k}(x)\right)\right| \leq C_{m}^{\prime} .
$$

Set $\varepsilon_{m}:=2^{-m} / C_{m}^{\prime}$. Then for any $k, \gamma$ and $x$,

$$
\sum_{|\alpha| \geq \max (k,|\gamma|)} W(x)\left|D^{\alpha}\left(x^{\gamma} \phi_{k}(x)\right)\right| \varepsilon_{|\alpha|} \leq \sum_{\alpha} 2^{-|\alpha|}=2^{n}<\infty,
$$

and the claim follows.

LEMMA 3. There exist positive constants $A_{k}, k \in \mathbb{N}$, such that $A_{k} \geq \gamma_{0, k}$ and

$$
A_{k} A_{|\iota|} \geq 2^{|\iota|}\left(\begin{array}{c}
\iota \\
\mu
\end{array}\right) \gamma_{\iota-\mu, k}\left(\begin{array}{l}
\mu \\
\pi
\end{array}\right)
$$

for any multiindices $\iota, \mu, \pi$ such that $\pi \subset \mu \subset \iota$.

Here we are using the usual multiindex notation

$$
\left(\begin{array}{l}
\iota \\
\mu
\end{array}\right):=\left(\begin{array}{l}
\iota_{1} \\
\mu_{1}
\end{array}\right) \cdots\left(\begin{array}{l}
\iota_{n} \\
\mu_{n}
\end{array}\right),
$$

and $\pi \subset \mu$ means that $\pi_{j} \leq \mu_{j}$ for all $j$. 
Proof. The right-hand side of (13) can be bounded by a constant $A(k, \iota)$ depending on $k$ and $\iota$ only. Take

$$
A_{m}:=\max _{k,|\iota| \leq m} A(k, \iota)+1 .
$$

It is easy to see that (13) follows.

We now define, inductively, a sequence of small positive numbers $a_{m, i}$, $m, i \in \mathbb{N}$. Assume that $m$ and $i$ are given and that $a_{k, j}$ has already been defined for all $(k, j)$ with $k<m$, or $k=m$ and $j<i$. We choose $a_{m, i}>0$ so small that the following requirements are satisfied:

$$
\begin{array}{ll}
a_{m, i} \leq \varepsilon_{i} \leq 1, & \\
a_{m, i} \leq a_{m-1, i} & \text { if } m \geq 1, \\
a_{m, i} \leq a_{m-1, i+1} & \text { if } m \geq 1, \\
a_{m, i} \leq \frac{a_{m, p} a_{m, q}}{A_{i}} & \text { for all } p, q<i .
\end{array}
$$

Lemma 4. For all $k, m \in \mathbb{N}$ and $\mu, \pi \in \mathbb{N}^{n}$ with $\pi \subset \mu$,

$$
\sum_{\iota \supset \mu} a_{m,|\iota|}\left(\begin{array}{l}
\iota \\
\mu
\end{array}\right) \gamma_{\iota-\mu, k}\left(\begin{array}{l}
\mu \\
\pi
\end{array}\right) \leq\left(2^{n}+1\right) A_{k} \frac{a_{m,|\mu-\pi|} a_{m,|\pi|}}{a_{m, 0}},
$$

where $A_{k}$ is as in (13).

Proof. As a combination of (13) and (17) we obtain

$$
\begin{aligned}
\sum_{\iota \supsetneq \mu} a_{m,|\iota|}\left(\begin{array}{l}
\iota \\
\mu
\end{array}\right) \gamma_{\iota-\mu, k}\left(\begin{array}{l}
\mu \\
\pi
\end{array}\right) & \leq A_{k} \sum_{\iota \supsetneq \mu} a_{m,|\iota|} A_{|\iota|} 2^{-|\iota|} \\
& \leq A_{k} a_{m,|\mu-\pi|} a_{m,|\pi|} \sum_{\iota \supsetneq \mu} 2^{-|\iota|} \\
& \leq 2^{n} A_{k} a_{m,|\mu-\pi|} a_{m,|\pi|} \leq 2^{n} A_{k} \frac{a_{m,|\mu-\pi|} a_{m,|\pi|}}{a_{m, 0}},
\end{aligned}
$$

since $a_{m, 0} \leq 1$ by construction. The remaining term $\iota=\mu$ reduces to $a_{m,|\mu|} \gamma_{0, k}\left(\begin{array}{c}\mu \\ \pi\end{array}\right)$. For $0<|\pi|<|\mu|$ we can again use (13) and (17) to bound this by $A_{k} a_{m,|\mu-\pi|} a_{m,|\pi|} 2^{-|\mu|} \leq A_{k} a_{m,|\mu-\pi|} a_{m,|\pi|}$. For $\pi=0$ or $\pi=\mu$, the term becomes just $a_{m,|\mu|} \gamma_{0, k}$, which is again bounded by $\frac{a_{m,|\mu|} a_{m, 0}}{a_{m, 0}} A_{k}$ since $A_{k} \geq \gamma_{0, k}$ by Lemma 3 .

We proceed to define the space $\mathcal{Z}:=\operatorname{ind}_{k \rightarrow \infty} \mathcal{Z}_{k}$. The step spaces $\mathcal{Z}_{k}$ are defined to consist of the $C^{\infty}$ functions $f$ on $\Omega$ such that

$$
\|f\|_{k}:=\sup _{x \in \Omega} W(x) w(x)^{-k} \sum_{\alpha} a_{k,|\alpha|}\left|D^{\alpha} f(x)\right|<\infty .
$$


We have $\mathcal{Z}_{k} \subset \mathcal{Z}_{k+1}$ continuously, for all $k$. In fact, $\|f\|_{k+1} \leq\|f\|_{k}$ by (15) and the fact that $w \geq 1$.

To recall the basic properties of the inductive limit, we have $\mathcal{Z}:=\bigcup_{k} \mathcal{Z}_{k}$, and if we define $U_{k}:=\left\{f \in C^{\infty}(\Omega):\|f\|_{k} \leq 1\right\}$, then a basis of neighbourhoods of zero is given by the sets

$$
U:=\Gamma \bigcup_{k=1}^{\infty} b_{k} U_{k}:=\left\{\sum_{k} \lambda_{k} g_{k}: \sum_{k}\left|\lambda_{k}\right| \leq 1, g_{k} \in b_{k} U_{k}\right\}
$$

for $b_{j}>0$. Here the sum in the definition of $U$ is allowed to have only a finite number of terms.

We are now going to prove that the space $\mathcal{Z}$ satisfies the conditions (a)-(e) from the preceding section. This will finish the proof of our Main Theorem.

Proof of (a). By Lemma 2 and (14), all functions of the form $p \phi_{k}$, where $k \in \mathbb{N}$ and $p$ is an arbitrary polynomial, belong to $\mathcal{Z}$. Since these functions obviously also belong to $\mathcal{D}$ and the germ of $p \phi_{k}$ at any point of $\operatorname{supp} \phi_{k-1}$ coincides with the germ of $p$ at that point, it is obvious that $\mathcal{D} \cap \mathcal{Z}$ is "large" in the sense of (8).

Proof of (b). It is enough to show that for each $l$ and $\alpha$, the operator $v_{l} D^{\alpha}$ maps $\mathcal{Z}$ into $L^{\infty}$. However, if $f \in \mathcal{Z}_{k}$, then

$$
\begin{aligned}
\left|v_{l}(x) D^{\alpha} f(x)\right| & \leq\left|v_{l}(x)\right| \frac{\|f\|_{k} w(x)^{k}}{a_{k,|\alpha|} W(x)} \leq \gamma_{0, l} \frac{\|f\|_{k}}{a_{k,|\alpha|}} \frac{w(x)^{k+1}}{W(x)} \\
& \leq \gamma_{0, l} \frac{\|f\|_{k}}{a_{k,|\alpha|}} \kappa_{k+1}<\infty
\end{aligned}
$$

for any $x \in \Omega$.

Proof of (c). Again, it is enough to prove that $v_{l}\left(D^{\alpha} f\right)\left(D^{\beta} g\right) \in L^{\infty}$ for any $l \in \mathbb{N}, \alpha, \beta \in \mathbb{N}^{n}$ and $f, g \in \mathcal{Z}$. However, for $f \in \mathcal{Z}_{k}$ and $g \in \mathcal{Z}_{m}$, by a similar argument to the one above,

$$
\begin{aligned}
\left|v_{l}\left(D^{\alpha} f\right)\left(D^{\beta} g\right)\right| & \leq \gamma_{0, l} w \frac{\|f\|_{k} w^{k}}{a_{k,|\alpha|} W} \frac{\|g\|_{m} w^{m}}{a_{m,|\beta|} W} \\
& \leq \gamma_{0, l} \frac{\|f\|_{k}\|g\|_{m}}{a_{k,|\alpha|} a_{m,|\beta|}} \kappa_{k+1} \kappa_{m}<\infty .
\end{aligned}
$$

Proof of $(d)$. Once more, we need only prove that $v_{k}\left(D^{\alpha} f\right)\left(D^{\beta} g\right)$ belongs to $\mathcal{Z}$ whenever $k \in \mathbb{N}, \alpha, \beta$ are multiindices and $f, g \in \mathcal{Z}$.

Assume that a neighbourhood of 0 as in (18) is given. So we are given a positive sequence $\left\{b_{j}\right\}_{j \in \mathbb{N}}$. 
We pick numbers $\widetilde{b}_{j}>0, j \in \mathbb{N}$, such that for all $j$ we have

$$
\widetilde{b}_{j} \leq \min _{m \leq 2 j+|\alpha|+|\beta|}\left(\frac{b_{m} a_{m, 0}}{\kappa_{m+1}\left(2^{n}+1\right) A_{k}}, 1\right) .
$$

We claim that $f \in \Gamma \bigcup_{j=1}^{\infty} \widetilde{b}_{j} U_{j}$ and $g \in \Gamma \bigcup_{j=1}^{\infty} \widetilde{b}_{j} U_{j}$ imply

$$
v_{k}\left(D^{\alpha} f\right)\left(D^{\beta} g\right) \in \Gamma \bigcup_{j=1}^{\infty} b_{j} U_{j}
$$

By the assumptions on $f$ and $g$, we can find finite sets of complex numbers $\lambda_{j}$ and $\mu_{j}$ and functions $f_{j} \in \widetilde{b}_{j} U_{j}$ and $g_{j} \in \widetilde{b}_{j} U_{j}$ such that

$$
\sum_{j}\left|\lambda_{j}\right| \leq 1, \quad \sum_{j}\left|\mu_{j}\right| \leq 1, \quad f=\sum_{j} \lambda_{j} f_{j}, \quad g=\sum_{j} \mu_{j} g_{j} .
$$

We have

$$
v_{k}\left(D^{\alpha} f\right)\left(D^{\beta} g\right)=\sum_{j, l} \lambda_{j} \mu_{l} v_{k}\left(D^{\alpha} f_{j}\right)\left(D^{\beta} g_{l}\right),
$$

and here $\sum_{j, l}\left|\lambda_{j} \mu_{l}\right| \leq 1$, so it is enough to prove that for all $j$ and $l$ there exists $m$ such that $v_{k}\left(D^{\alpha} f_{j}\right)\left(D^{\beta} g_{l}\right) \in b_{m} U_{m}$.

We claim that this happens for $m:=j+l+|\alpha|+|\beta|$. Recall that $\left|D^{\iota} v_{k}\right| \leq$ $\gamma_{\iota, k} w$ by Lemma 1 .

We have

$$
\begin{aligned}
& \left\|v_{k}\left(D^{\alpha} f_{j}\right)\left(D^{\beta} g_{l}\right)\right\|_{m} \\
& \quad=\sup _{x \in \Omega} W(x) w(x)^{-m} \sum_{\iota} a_{m,|\iota|}\left|D^{\iota}\left(v_{k}\left(D^{\alpha} f_{j}\right)\left(D^{\beta} g_{l}\right)\right)\right| .
\end{aligned}
$$

By the Leibniz rule,

$$
\begin{aligned}
\sum_{\iota} a_{m,|\iota|}\left|D^{\iota}\left(v_{k}\left(D^{\alpha} f_{j}\right)\left(D^{\beta} g_{l}\right)\right)\right| \\
\leq \sum_{\iota} a_{m,|\iota|} \sum_{\mu \subset \iota}\left(\begin{array}{l}
\iota \\
\mu
\end{array}\right)\left|\left(D^{\iota-\mu} v_{k}\right) D^{\mu}\left(\left(D^{\alpha} f_{j}\right)\left(D^{\beta} g_{l}\right)\right)\right| \\
\leq \sum_{\iota} a_{m,|\iota|} \sum_{\mu \subset \iota}\left(\begin{array}{l}
\iota \\
\mu
\end{array}\right) \gamma_{\iota-\mu, k} w \sum_{\pi \subset \mu}\left(\begin{array}{c}
\mu \\
\pi
\end{array}\right)\left|\left(D^{\alpha+\mu-\pi} f_{j}\right)\left(D^{\beta+\pi} g_{l}\right)\right| \\
\quad=w \sum_{\pi} \sum_{\mu \supset \pi} \sum_{\iota \supset \mu} a_{m, \iota \iota}\left(\begin{array}{c}
\iota \\
\mu
\end{array}\right) \gamma_{\iota-\mu, k}\left(\begin{array}{c}
\mu \\
\pi
\end{array}\right)\left|\left(D^{\alpha+\mu-\pi} f_{j}\right)\left(D^{\beta+\pi} g_{l}\right)\right| .
\end{aligned}
$$

By Lemma 4 this is bounded by

$$
\frac{\left(2^{n}+1\right) A_{k}}{a_{m, 0}} w \sum_{\pi} \sum_{\mu \supset \pi} a_{m,|\mu-\pi|} a_{m,|\pi|}\left|\left(D^{\alpha+\mu-\pi} f_{j}\right)\left(D^{\beta+\pi} g_{l}\right)\right| .
$$


Hence, (20) can be bounded by

$$
\begin{aligned}
\frac{\left(2^{n}+1\right) A_{k}}{a_{m, 0}} \sup _{x \in \Omega} W(x) w(x)^{-m+1} & \times \sum_{\pi} \sum_{\mu \supset \pi} a_{m,|\mu-\pi|} a_{m,|\pi|}\left|\left(D^{\alpha+\mu-\pi} f_{j}\right)\left(D^{\beta+\pi} g_{l}\right)\right| \\
\leq & \frac{\left(2^{n}+1\right) A_{k}}{a_{m, 0}}\left(\sup _{\Omega} W w^{-m} \sum_{\mu} a_{m,|\mu|}\left|D^{\alpha+\mu} f_{j}\right|\right) \\
& \times\left(\sup _{\Omega} W w^{-m} \sum_{\mu} a_{m,|\mu|}\left|D^{\beta+\mu} g_{l}\right|\right) \cdot\left(\sup _{\Omega} \frac{w^{m+1}}{W}\right) \\
\leq & \kappa_{m+1} \frac{\left(2^{n}+1\right) A_{k}}{a_{m, 0}}\left(\sup _{\Omega} W w^{-m+|\alpha|} \sum_{\mu} a_{m-|\alpha|,|\mu+\alpha|}\left|D^{\alpha+\mu} f_{j}\right|\right) \\
& \times\left(\sup _{\Omega} W w^{-m+|\beta|} \sum_{\mu} a_{m-|\beta|,|\mu+\beta|}\left|D^{\beta+\mu} g_{l}\right|\right) \quad \text { by }(16) \text { and } w \geq 1 \\
\leq & \kappa_{m+1} \frac{\left(2^{n}+1\right) A_{k}}{a_{m, 0}}\left(\sup _{\Omega} W w^{-m+|\alpha|} \sum_{\gamma} a_{m-|\alpha|,|\gamma|}\left|D^{\gamma} f_{j}\right|\right) \\
& \times\left(\sup _{\Omega} W w^{-m+|\beta|} \sum_{\gamma} a_{m-|\beta|,|\gamma|}\left|D^{\gamma} g_{l}\right|\right) \\
\leq & \left(2^{n}+1\right) A_{k} \frac{\kappa_{m+1}}{a_{m, 0}} \widetilde{b}_{j} \widetilde{b}_{l} \leq b_{m} .
\end{aligned}
$$

Here in the penultimate inequality we have used the fact that

$$
\sup _{\Omega} W w^{-m+|\alpha|} \sum_{\gamma} a_{m-|\alpha|,|\gamma|}\left|D^{\gamma} f_{j}\right|=\left\|f_{j}\right\|_{m-|\alpha|} \leq\left\|f_{j}\right\|_{j} \leq \widetilde{b}_{j},
$$

since $m-|\alpha|=j+l+|\beta| \geq j$ (and similarly for $g_{l}$ ); while the very last inequality follows from (19).

Proof of (e). Let us first of all remark that $L_{b}\left(\mathcal{Z}, L^{\infty}(\Omega)\right)$ is indeed a Fréchet space. Indeed, since every $(L B)$-space is a $(D F)$-space (cf. [Koe, part I, §29.5(5)]), and $L_{b}(E, F)$ is complete if $E$ is $(D F)$ and $F$ is any complete locally convex space (see [Koe, part II, §39.6(6)]), the completeness of $L_{b}\left(\mathcal{Z}, L^{\infty}(\Omega)\right)$ follows. As for metrizability, $\mathcal{Z}$ has a fundamental sequence $\left(B_{n}\right)_{n=1}^{\infty}$ of bounded sets (by construction, or by the fact that it is $(D F)$, see [Koe, part I, beginning of §29.3]). Hence every bounded subset of $\mathcal{Z}$ is contained in some multiple $c B_{n}$, and from the definition of the topology of uniform convergence on bounded sets we see that the countably many sets

$$
\mathcal{U}_{n}:=\left\{T \in L_{b}\left(\mathcal{Z}, L^{\infty}\right):\|T f\|_{\infty} \leq 1 \text { for all } f \in B_{n}\right\}
$$


form a basis of neighbourhoods of 0 in $L_{b}\left(\mathcal{Z}, L^{\infty}\right)$. Thus $L_{b}\left(\mathcal{Z}, L^{\infty}\right)$ is Fréchet.

Let us now show in detail how the norm estimate (e) follows from Colombeau's result. For the various facts from the differential calculus in Banach spaces, we refer to [Cha].

Recall that a continuous mapping $P: Y \rightarrow X$, where $Y$ and $X$ are Banach spaces, is called a (continuous) $j$-homogeneous polynomial if there exists a continuous symmetric $j$-linear mapping $\widetilde{P}: Y^{j} \rightarrow X$ such that $P(x)=\widetilde{P}(x, \ldots, x)$. The definition for locally convex spaces is the same. Given a $P$ as above, the mapping $\widetilde{P}$ is unique and can be constructed from $P$ using the polarization formula ([Cha, Theorems 4.6 and 4.7]).

For all $j \in \mathbb{N}$ let us denote by $N_{j}$ the $j$-homogeneous polynomial

$$
N_{j}(h):=h^{j} M_{j}
$$

from $\mathbb{R}$ into $L_{b}\left(\mathcal{Z}, L^{\infty}(\Omega)\right)=: X$. Let $\widetilde{N}_{j}$ be the corresponding symmetric $j$-linear mapping. Clearly, both $N_{j}$ and $\tilde{N}_{j}$ are continuous.

By the main theorem of [Col] we find a $C^{\infty}$-mapping $M: \mathbb{R} \rightarrow X$ whose $n$th derivative coincides with $\widetilde{N}_{j}$. By the explanation after the main theorem in [Col], the $j$ th derivative of $M$ is even a continuous $j$-linear mapping from $\mathbb{R}$ to $X_{B}$, where $X_{B} \subset X$ denotes the linear span of some bounded convex balanced subset $B \subset X$. Also, $X_{B}$ is a Banach space when endowed with the norm $\|x\|_{B}:=1 / \sup \{r>0: r x \in B\}$, and the canonical injection $X_{B} \rightarrow X$ is continuous.

Hence, also $\tilde{N}_{j}: \mathbb{R}^{j} \rightarrow X_{B}$ is continuous. By the Taylor formula for Banach spaces ([Cha, Theorem 8.9]), we obtain the error estimate $\left(M^{(h)}=M(h)\right)$

$$
\left\|M^{(h)}-\sum_{j=0}^{N} h^{j} M_{j}\right\|_{B} \leq C_{N} h^{N+1} .
$$

Now every bounded set $B \subset X$ is contained in the set

$$
U^{\circ}:=\left\{T \in X:\|T f\|_{\infty} \leq 1 \text { for all } f \in U\right\}
$$

for some neighbourhood of zero $U$ of $\mathcal{Z}$. Hence,

$$
\left\|M^{(h)}-\sum_{j=0}^{N} h^{j} M_{j}\right\|_{B} \geq \sup _{f \in U}\left\|\left(M^{(h)}-\sum_{j=0}^{N} h^{j} M_{j}\right) f\right\|_{\infty} .
$$

Let now $f \in Z$ be given. Since $U$ is a neighbourhood of 0 , it absorbs $f$, i.e. there exists a $c>0$ such that $f \in c U$. The desired norm estimate (e) (for some $f$-dependent constant) follows from this, (21) and (23).

This completes the proof of the Main Theorem. 
REMARK. Note that in the above proofs we have actually established assertions slightly stronger than the properties (b)-(d), namely that the inclusions given there are in fact continuous: that is, for each $j, k$ and $l$, $M_{j}$ maps $\mathcal{Z}$ continuously into $L^{\infty}, C_{l}\left(M_{j} \cdot, M_{k} \cdot\right)$ maps $\mathcal{Z} \times \mathcal{Z}$ continuously into $L^{\infty}$, and $C_{l}^{\prime}$ even maps $\mathcal{Z} \times \mathcal{Z}$ continuously into $\mathcal{Z}$. (Well, for (b) and (c), we have only shown that the operators map bounded sets into balls, but this implies continuity by a simple argument: one forms a small enough neighbourhood $U$ of 0 in $\mathcal{Z}$ using just the definition of the inductive limit topology - that is, $U$ is chosen as the balanced convex hull of the union of countably many bounded sets (the unit balls of the step spaces) multiplied by small constants; if the constants are small enough, $U$ is still mapped into the unit ball of $L^{\infty}$.) However, this extra piece of information seems to have no implications for the applications to quantization.

4. A nonlinear variant. Note that the argument (15) still works even if the operators $M^{(h)}$ and $Q^{(h)}$ are not assumed to be linear, i.e. if we just require that for each fixed $f \in \mathcal{Z}$ there be some functions $M^{(h)} f \in L^{\infty}$, $0<h<1$, such that (e) holds. In that case, the sought-for operators $M^{(h)}$ can be constructed for $\mathcal{Z}=\mathcal{D}$ directly along the lines of the usual proof of Borel's theorem. Let us include a proof of this assertion for completeness.

Take $\mathcal{Z}=\mathcal{D}(\Omega)$, the subspace of functions in $C^{\infty}(\Omega)$ with compact support. Then clearly (a)-(d) hold; let us settle (e) (allowing $M^{(h)}$ to be nonlinear). So let $f \in \mathcal{D}$, and define for brevity $m_{j}=M_{j} f \in \mathcal{D}$.

Fix a function $\phi \in C^{\infty}(\mathbb{R})$ such that $0 \leq \phi \leq 1$ and $\phi(x)=1$ for $|x| \leq 1 / 2, \phi(x)=0$ for $|x| \geq 1$. Let $\nu_{n, \alpha}:=\left\|D^{\alpha} m_{n}\right\|_{\infty}$ and set $q_{j}:=$ $\max _{|\alpha|, n \leq j} \nu_{\alpha, n}+1$, so that $\nu_{n, \alpha} \leq q_{n} q_{|\alpha|}$. Let now $c_{n}:=\max \left(2, n ! q_{n}\right)$ and define

$$
u_{n}(x, h)=\phi\left(c_{n} h\right) m_{n}(x) h^{n} .
$$

Clearly each $u_{n}$ is a bounded smooth function on $\Omega \times \mathbb{R}$. Observe that $u_{n}$ is nonzero only if

$$
|h| \leq 1 / c_{n} \leq 1 / 2,
$$

owing to the fact that $c_{n} \geq 2$. Using the Leibniz rule, for any integer $k \geq 0$ and multiindex $\alpha$ we have

$$
D_{h}^{k} D_{x}^{\alpha} u_{n}(x, h)=D^{\alpha} m_{n}(x) \cdot \sum_{j=0}^{k}\left(\begin{array}{c}
k \\
j
\end{array}\right) D^{k-j} h^{n} \cdot c_{n}^{j} \phi^{(j)}\left(c_{n} h\right),
$$

where the subscripts at $D$ indicate the differentiated variable. The last factor on the right is bounded by $|h|^{-j} s_{j}$ where $s_{j}:=\sup _{x \in \mathbb{R}}\left|x^{j} \phi^{(j)}(x)\right|$ $<\infty$. Moreover, $D^{k-j} h^{n}=(n ! /(n-k+j) !) h^{n-k+j}$, which is bounded by 
$n !|h|^{n-k+j}$ (and vanishes for $\left.j<k-n\right)$. Thus

$$
\begin{aligned}
\left|D_{h}^{k} D_{x}^{\alpha} u_{n}(x, h)\right| & \leq\left\|D^{\alpha} m_{n}\right\|_{\infty} \sum_{j=0}^{k}\left(\begin{array}{l}
k \\
j
\end{array}\right) n !|h|^{n-k} s_{j} \\
& \leq q_{n} q_{|\alpha|} n !|h|^{n-k} W_{k}
\end{aligned}
$$

where $W_{k}:=\sum_{j=0}^{k}\left(\begin{array}{c}k \\ j\end{array}\right) s_{j}<\infty$. Since $c_{n} \geq n ! q_{n}$, it follows that

$$
\begin{aligned}
\sum_{n=k+1}^{\infty}\left|D_{h}^{k} D_{x}^{\alpha} u_{n}(x, h)\right| & \leq q_{|\alpha|} W_{k} \sum_{n=k+1}^{\infty} q_{n} n !|h|^{n-k} \\
& \leq q_{|\alpha|} W_{k} \sum_{n=k+1}^{\infty} \frac{q_{n} n !}{c_{n}}|h|^{n-k-1} \\
& \leq 2 q_{|\alpha|} W_{k}<\infty
\end{aligned}
$$

As $\alpha$ and $k$ can be arbitrary, we see that the series

$$
u(x, h):=\sum_{n=0}^{\infty} u_{n}(x, h)
$$

converges in the $C^{\infty}$ topology to a function $u \in C^{\infty}(\Omega \times \mathbb{R})$. Further, as each $u_{n}$ is, in view of (24), supported in supp $m_{n} \times[-1 / 2,1 / 2] \subset \operatorname{supp} f \times$ $[-1 / 2,1 / 2]$, we even have $u \in \mathcal{D}(\Omega \times(-1,1))$. Since

$$
D_{h}^{j} u_{n}(x, 0)= \begin{cases}0 & \text { if } j \neq n \\ j ! m_{j}(x) & \text { if } j=n\end{cases}
$$

the $C^{\infty}$ convergence of $(25)$ implies that $D_{h}^{j} u(x, 0)=j ! m_{j}(x)$. By the Taylor remainder formula, we therefore have, for any integer $N \geq 0$,

$$
u(x, h)-\sum_{j=0}^{N} m_{j}(x) h^{j}=\frac{h^{N+1}}{(N+1) !} D_{h}^{N+1} u(x, \theta(x, h) h)
$$

for some $0 \leq \theta(x, h) \leq 1$; consequently,

$$
h^{-N-1}\left|u(x, h)-\sum_{j=0}^{N} m_{j}(x) h^{j}\right| \leq \frac{1}{(N+1) !}\left\|D_{h}^{N+1} u\right\|_{\infty}<\infty, \quad \forall x, h,
$$

since $u$ is compactly supported. Thus the choice

$$
M^{(h)} f(x):=u(x, h)
$$

will do the job we need.

REMARK. In effect, the above argument proves Borel's theorem for functions from $\mathbb{R}$ into $\mathcal{D}$, for the case that the prescribed derivatives $m_{j}$ have supports in a fixed compact set. Perhaps there is some hope that Borel's 
theorem might hold even for functions from $\mathbb{R}$ into more general locally convex spaces provided some additional hypothesis is assumed on the sequence of the prescribed derivatives - for instance, if the target space is a space of operators, when $M_{j}$ are "tame" in the sense that there exists a shrinking collection $U_{k}$ of neighbourhoods of zero such that each $M_{j}$ maps $U_{k+1}$ into $U_{k}$, for all $k$. Note that having larger and larger supports is also the idea behind Colombeau's counterexample [Col] showing that Borel's theorem fails for functions from $\mathbb{R}$ into $\mathcal{D}$.

It should be remarked that from the point of view of physics, the nonlinear quantization treated in this section is probably a rather doubtful business.

\section{References}

[AE] S. T. Ali and M. Engliš, Quantization methods: a guide for physicists and analysts, Rev. Math. Phys. 17 (2005), 391-490.

[BF] F. Bayen, M. Flato, C. Fronsdal, A. Lichnerowicz and D. Sternheimer, Deformation theory and quantization, Lett. Math. Phys. 1 (1977), 521-530; Ann. Phys. 111 (1978), 61-110 (part I), 111-151 (part II).

[BnC] J. Bonet and P. Pérez Carreras, Barrelled Locally Convex Spaces, North-Holland Math. Stud. 131, North-Holland, Amsterdam, 1987.

[BMS] M. Bordemann, E. Meinrenken and M. Schlichenmaier, Toeplitz quantization of Kähler manifolds and $g l(n), n \rightarrow \infty$ limits, Comm. Math. Phys. 165 (1994), 281-296.

[BLU] D. Borthwick, A. Leśniewski and H. Upmeier, Non-perturbative deformation quantization on Cartan domains, J. Funct. Anal. 113 (1993), 153-176.

[Cha] S. B. Chae, Holomorphy and Calculus in Normed Spaces, Dekker, New York, 1985.

[Cob] L. A. Coburn, Berezin-Toeplitz quantization, in: Algebraic Methods in Operator Theory, Birkhäuser, 1994, 101-108.

[Col] J. F. Colombeau, Infinite dimensional $C^{\infty}$ mappings with a given sequence of derivatives at a given point, J. Math. Anal. Appl. 71 (1979), 95-104.

[E1] M. Engliš, Weighted Bergman kernels and quantization, Comm. Math. Phys. 227 (2002), 211-241.

[E2] - Berezin-Toeplitz quantization and invariant symbolic calculi, Lett. Math. Phys. 65 (2003), 59-74.

[E3] - Berezin-Toeplitz quantization on the Schwartz space of bounded symmetric domains, J. Lie Theory 15 (2005), 27-50.

[Gu] S. Gutt, Variations on deformation quantization, in: Conférence Moshé Flato (Dijon, 1999), Vol. I, Math. Phys. Stud. 21, Kluwer, Dordrecht, 2000, 217-254.

[Ja] H. Jarchow, Locally Convex Spaces, Teubner, Stuttgart, 1981.

[KL] S. Klimek and A. Leśniewski, Quantum Riemann surfaces, I: The unit disc, Comm. Math. Phys. 146 (1992), 103-122.

[Koe] G. Köthe, Topological Vector Spaces I, II, Springer, Berlin, 1969, 1979.

[MV] R. Meise and D. Vogt, Introduction to Functional Analysis, Oxford Univ. Press, New York, 1997. 
[Sch] M. Schlichenmaier, Deformation quantization of compact Kähler manifolds by Berezin-Toeplitz quantization, in: Conférence Moshé Flato 1999 (Dijon, 1999), Vol. 2, Kluwer, 2000, 289-306.

Mathematics Institute

Žitná 25

11567 Praha 1, Czech Republic

and

Mathematics Institute

Na Rybníčku 1

74601 Opava, Czech Republic

E-mail: englis@math.cas.cz
Department of Mathematics and Statistics University of Helsinki

P.O. Box 68 FIN-00014 Helsinki, Finland E-mail: jari.taskinen@helsinki.fi

Received March 31, 2006

Revised version February 18, 2007 